\title{
An Eight-Channel Switching-Linear Hybrid Dynamic Regulator with Dual-Supply LDOs for Thermo-Optic Tuning
}

This paper was downloaded from TechRxiv (https://www.techrxiv.org).

\section{LICENSE}

CC BY-NC-SA 4.0

SUBMISSION DATE / POSTED DATE

$13-02-2022$ / 22-02-2022

\section{CITATION}

Zhang, Siyuan; Wang, Xingze; tan, min (2022): An Eight-Channel Switching-Linear Hybrid Dynamic Regulator with Dual-Supply LDOs for Thermo-Optic Tuning. TechRxiv. Preprint. https://doi.org/10.36227/techrxiv.19165589.v1

DOI 


\title{
An Eight-Channel Switching-Linear Hybrid Dynamic Regulator with Dual-Supply LDOs for Thermo-Optic Tuning
}

\author{
Siyuan Zhang, Student Member, IEEE, Ken Xingze Wang and Min Tan*, Member, IEEE
}

\begin{abstract}
A novel switching-linear hybrid dynamic regulator architecture with dual-supply low dropout regulators (LDOs) is presented in this paper. This architecture leverages the intrinsic dual supplies to extend the operating range of the LDOs. Furthermore, it increases the thermo-optic tuning efficiency by reducing the LDO dropout voltage through dynamic supply modulation. This architecture is suitable for large-scale thermooptic tuning in silicon photonics. The efficiency improvement is particularly effective when tracking signals of several adjacent channels are close to each other, e.g., wavelength tuning of a microring array. The principle of this architecture is general and can be implemented using different switching converters and LDOs. A specific design with extensive post-layout simulation results is used to verify the effectiveness of our architecture. Implemented in a $130 \mathrm{~nm}$ CMOS process, this design can simultaneously regulate eight output channels with an output swing of $0.8 \mathrm{~V}_{\mathrm{pp}}$. Its peak efficiency when driving $100 \Omega$ loads is $92 \%$ at $1 \mathrm{~V}$ output, and the dynamic efficiency is around $86 \%$ when tracking $50 \mathrm{kHz}$ sinusoidal signals. To the best of our knowledge, this is the first time that dynamic supply modulation has been applied to the thermo-optic tuning of silicon photonic devices.
\end{abstract}

Index Terms - Thermo-optic tuning, hybrid dynamic regulator, dual-supply LDO, multiple output channels, envelope tracking.

\section{INTRODUCTION}

$\mathbf{S}$ ILICON photonics $(\mathrm{SiPh})$ is attracting increasing attention in recent years [1], [2], and is an enabling technology for emerging applications such as optical phased arrays (OPA) [3], [4], silicon quantum photonic circuits [5], [6], and optical neural networks (ONN) [7].

Thermo-optic tuning is an effective dynamic control technique for integrated photonic devices and has been extensively used in many important applications, including OPA [4], [9], [10], [14], ONN [7], thermo-optic phase shifters [8], wavelength tuning of a microring modulator [11]-[13], etc. The thermo-optic tuning dynamically adjusts the effective refractive index of photonic devices by changing the temperature, and a general model for closed-loop thermo-optic feedback tuning is shown in Fig. 1. The power management circuits (PMCs) regulate the supply voltage on the purely resistive heaters based on the input reference signals, i.e., $V_{\mathrm{RHi}}$ changes according to $V_{\mathrm{REFi}}(\mathrm{i}=1,2, \ldots, \mathrm{n})$. By regulating the supply of different heaters, the temperature of different

Siyuan Zhang and Min Tan are with the School of Optical and Electronic Information, Huazhong University of Science and Technology, Wuhan 430074, China (email: mtan@hust.edu.cn).

Ken Xingze Wang is with the School of Physics, Huazhong University of Science and Technology, Wuhan 430074, China.

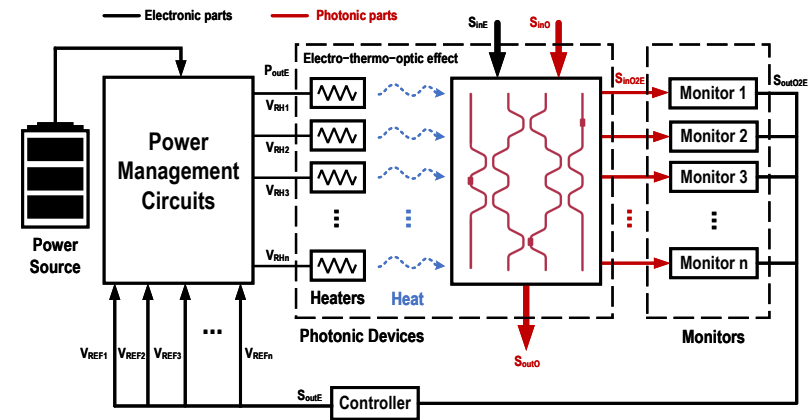

Fig. 1. The general model for close-loop thermo-optic feedback tuning [2].

photonic devices is adjusted separately. In large-scale $\mathrm{SiPh}$ integration, hundreds or even thousands of heaters need to be regulated simultaneously [4]-[10].

For large-scale and energy-efficient thermo-optic tuning, a multi-channel high-performance PMC with compact chip area is required. Most recent designs [5], [7], [8] apply PCB or benchtop solutions to tune photonic devices. This approach occupies a large off-chip area that limits the system scalability and is also power-hungry. Therefore, PCB or benchtop solutions are not suitable for large-scale $\mathrm{SiPh}$ integration. Many PMCs solutions have been proposed in the literature. Linear regulator is widely used in [11]-[13], the nonlinear current DAC is employed in [4], and the pulse width modulation (PWM) driving circuit is applied in [14].

The linear regulator occupies a relatively small on-chip area and has excellent scalability. However, the conventional linear regulator usually adopts a fixed supply voltage and cannot always keep high conversion efficiency in a wide tuning range. Meanwhile, the heat loss on PMC may also cause thermal crosstalk that interferes with other photonic devices. As shown in Fig. 2, we use wavelength tuning of a microring resonator (MRR) to illustrate the operation of thermo-optic tuning. The linear regulator in Fig. 2 (a)(b) dynamically changes its output power to maintain the MRR's wavelength. When the $V_{\mathrm{RH}}$ decreases, the conversion efficiency of the linear regulator with a fixed supply voltage $V_{\mathrm{DD}}$ will significantly drop. Alternatively, using a dynamically changing supply voltage in Fig. 2 (b) can reduce the dropout, therefore improving the power conversion efficiency of the linear regulator.

Dynamic supply modulation has been traditionally employed in radio-frequency power amplifiers (RF PA) [15][18] and audio-frequency power amplifiers (AF PA) [19]. 


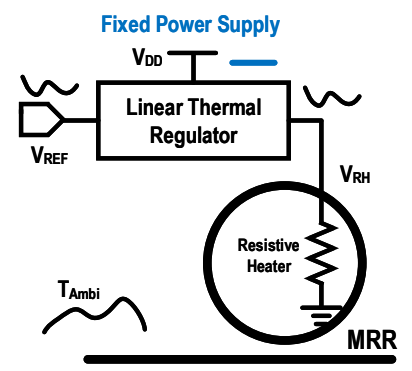

(a)

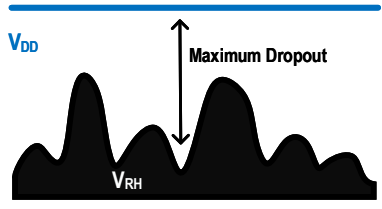

(c)

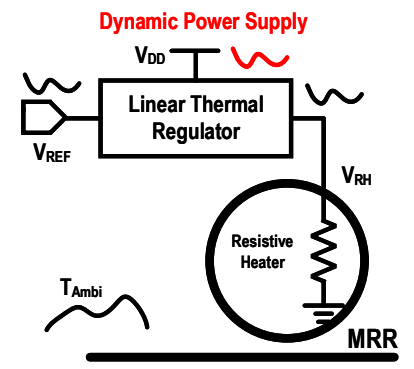

(b)

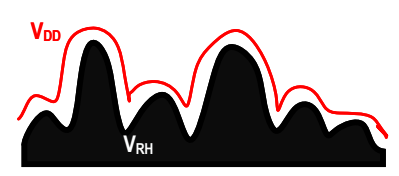

(d)
Fig. 2. Wavelength tuning of an MRR with (a) fixed supply voltage and (b) dynamically changing supply voltage; Voltage waveforms under (c) fixed supply voltage and (d) dynamically changing supply voltage.

TABLE I

COMPARISON BETWEEN THREE TYPES OF DYNAMIC SUPPLy MODULATION

\begin{tabular}{|c|c|c|c|}
\hline Application & Output Power & Envelope Tracking & Number of Channels \\
\hline RF PA & $\sim$ W & $\geq 10 \mathrm{MHz}$ & Single \\
\hline AF PA & $\sim$ W & $20 \sim 40 \mathrm{kHz}$ & Single \\
\hline $\begin{array}{c}\text { Thermo-Optic } \\
\text { Tuning }\end{array}$ & $\sim \mathrm{mW}$ & $\leq 100 \mathrm{kHz}$ & Multiple \\
\hline
\end{tabular}

However, the dynamic supply modulation for thermo-optic tuning differs greatly from the above applications in terms of power range, tracking speed, and the number of channels. TABLE I compares the differences among these three types of dynamic supply modulation. Because of this, the previous designs for RF/AF PA cannot be directly applied to thermooptic tuning.

In this paper, we present a novel switching-linear hybrid dynamic regulator architecture with dual-supply LDOs. The proposed architecture achieves both a high conversion efficiency and a wide tuning range, and is suitable for large-scale thermo-optic tuning. The remainder of this paper is organized as follows. Section II discusses the PMC requirements for large-scale thermo-optic tuning. Section III discusses one specific application of the proposed architecture. Section IV presents the structure and operating principles of a proposed design. Section V shows the verification results. Section VI draws the conclusion.

\section{PMC REQUIREMENTS FOR LARGE-SCALE THERMO-Optic TUNING}

After analyzing several different photonic devices tuned by the thermo-optic effect [20]-[24], we summarize the PMC requirements for thermo-optic tuning:

1) Equivalent load: the load in thermo-optic tuning is different from that in traditional applications. In traditional applications, the load of the PMC is random, and the transient response is regarded as an important indicator of the PMC performance. However, since the heater embedded in photonic device is essentially a fixed resistive load, we do not have to consider the transient response in thermo-optic tuning. We are more concerned with the tracking performance of the PMCs.

2) Tracking speed: the typical bandwidth of the photonic devices is around tens of $\mathrm{kHz}$. On the one hand, this requires the tracking speed of PMC to reach tens of $\mathrm{kHz}$ as well. On the other hand, since the temperature is mainly determined by the low-frequency average output power in thermo-optic tuning, the high-frequency noise will not affect the performance [25].

3) Output power range: the required output power for different photonic devices ranges from a few to tens of $\mathrm{mW}$. However, the total system power consumption cannot be ignored for large-scale $\mathrm{SiPh}$ integration. For instance, an integrated platform that requires 10,000 photonic devices, each consuming $10 \mathrm{~mW}$ of power, will require $100 \mathrm{~W}$ of power, which is prohibitively large for many applications.

4) Conversion efficiency: it is defined to evaluate the energy loss on the PMCs. The energy loss of the conventional linear regulator is close to or even larger than its output power in most conditions. In a large-scale integrated system, even a few percent improvement in system efficiency will save a lot of energy.

5) Average area per channel: it is defined as the average area required for the PMCs to tune a single photonic device, and can be written as the ratio of the PMCs' total area to the number of the channels. Generally, each channel in PMCs corresponds to a single photonic device. Since the size of a photonic device is relatively small, the matched PMC should not occupy too much area.

\section{Wavelength Tuning of Microring ArRay}

The efficiency improvement of the proposed architecture is particularly effective when the tracking signals of several adjacent channels are close to each other, e.g., power supply for wavelength tuning of microring array.

Compared with the single microring, the microring array has more application scenarios and is expected to achieve better performance. However, the resonant wavelength $\left(\lambda_{0}\right)$ of microrings is highly sensitive to thermal fluctuations and process variations. We need to maintain the $\lambda_{0}$ and guarantee microrings' stable operation by thermal-optic tuning through embedded heaters. The proposed architecture can be used to regulate multiple heaters simultaneously. For instance, one application of the microring array is the high-order seriescoupled MRR filter for better flat-top response and higher out-of-band signal rejection. All microrings of the high-order filter should be resonant at the same $\lambda_{0}$ [26]. The adjacent silicon microrings experience $0.15-0.55 \mathrm{~nm}$ of $\lambda_{0}$ variations from random process variations [27], [28] and $0.05-0.1 \mathrm{~nm} / \mathrm{K}$ of thermo-optic $\lambda_{0}$ shifts [29], [30]. Moreover, since the integrated heaters with efficiency $>1 \mathrm{~nm} / \mathrm{mW}$ have been demonstrated [11], [23], [24], [31], the heating powers required for several adjacent microrings to compensate for the wavelength variations are close to each other (the power difference can be less than $1 \mathrm{~mW}$ ). Meanwhile, as the heating power is 


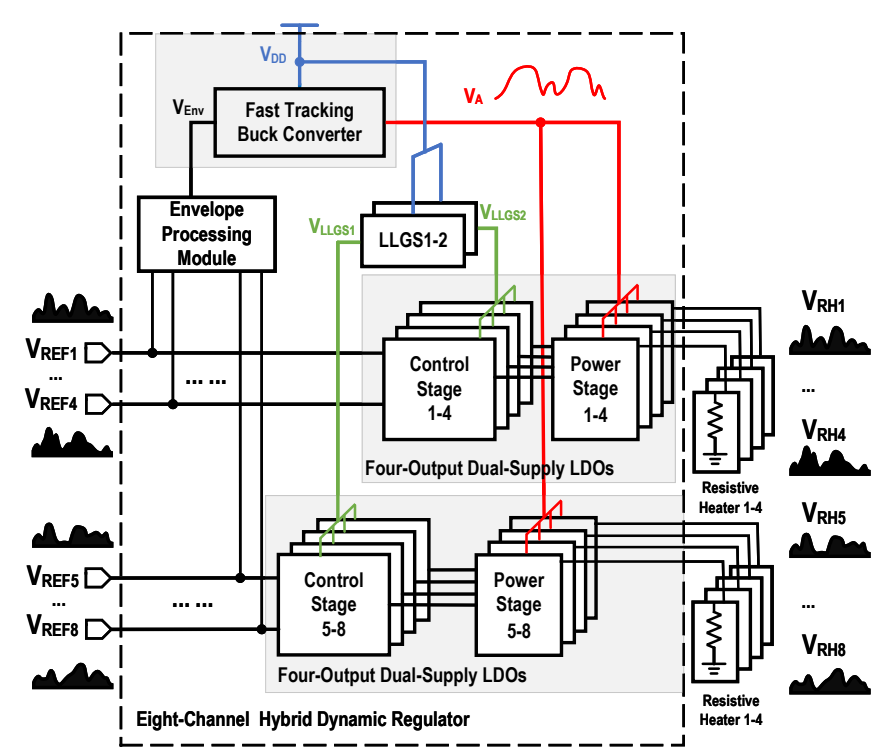

Fig. 3. Architecture of the proposed switching-linear hybrid dynamic regulator with dual-supply LDOs.

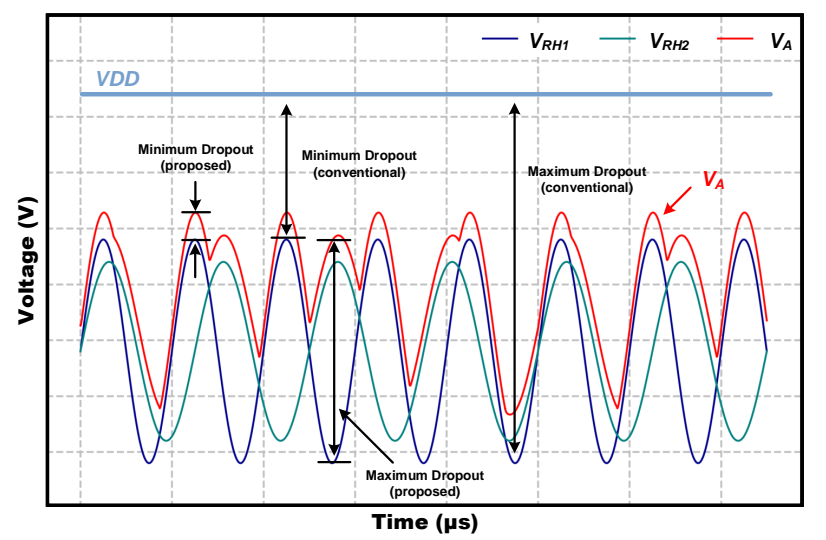

Fig. 4. Key waveforms of the dual-channel regulator with the same architecture.

proportional to the square of the PMC's output voltage, the tracking signals of several PMCs' adjacent channels are also close to each other. The proposed architecture is particularly useful for applications like this.

\section{EIGHT-CHANNEL HyBRID DYNAMIC REGULATOR}

In this section, we first demonstrate the proposed architecture through an eight-channel design. Then we present the structure and operating principles of this design. After that, we evaluate the conversion efficiency. Notice that the proposed architecture is not limited to one specific design, but can be implemented using different switching converters and LDOs.

\section{A. Proposed Architecture}

The architecture of the proposed multi-channel switchinglinear hybrid dynamic regulator with dual-supply LDOs is shown in Fig. 3. It has intrinsic dual supplies, i.e., the global supply $V_{\mathrm{DD}}$ and the intermediate supply $V_{\mathrm{A}}$, and mainly consists of an envelope processing module, a switching converter, and multiple parallel dual-supply LDOs. The LDOs are used to drive the heaters directly, and their control stages and power stages are supplied separately. The envelope processing module assists the switching converter to generate a suitable voltage $V_{\mathrm{A}}$ to supply LDOs' power stages. $V_{\mathrm{A}}$ should be as close as possible to the envelope of the LDOs' output voltages, but ensure the normal operation of their power stages. The architecture dynamically adjusts $V_{\mathrm{A}}$ according to the changes of the output voltage of the envelope processing module $V_{\mathrm{Env}}$, thereby reducing the LDOs' dropout voltage and improving the conversion efficiency. Besides, since $V_{\mathrm{A}}$ is not directly used as the output, the accuracy requirement for $V_{\mathrm{Env}}$ is relaxed, and small errors are allowed.

In our specific design, we combine a current-mode TypeIII buck converter and eight dual-supply LDOs with NMOS power transistors to verify the proposed architecture. We also introduce the lightweight local generated supply (LLGS) structure to provide an appropriate local voltage $V_{\text {LLGS }}$ for the control stages of the LDOs. However, the LLGS is not a necessary component of the proposed architecture, we can also supply the control stages with the global voltage $V_{\mathrm{DD}}$.

To help understand the operation principle of the proposed architecture, we provide the key waveforms of the dual-channel case with the same architecture in Fig. 4. The maximum and minimum dropouts in the proposed and the conventional design are compared in Fig. 4 as well.

\section{B. Dual-Supply LDO}

Fig. 5 shows the schematic of the dual-supply LDO. The proposed architecture has two intrinsic power supplies, one of them being the global power supply and the other the output voltage of the switching converter. The control stage of the LDO is a two-stage amplifier using current-mirror Miller (CMM) compensation [32]. To improve the LDO's PSR, the NMOS power transistor is employed.

The LDO mainly consists of the control stage (usually an error amplifier) and the power stage. To guarantee the stability of the negative feedback loop, the supply voltage of the control stage has to be sufficiently high to ensure the associated transistors can operate in the saturation region. However, to obtain high efficiency, when the output voltage dynamically changes in a wide range, the dropout voltage between the supply and the output of LDO should keep as small as possible at all times. Since the control and the power stage of the conventional LDO share the same global voltage $V_{\mathrm{DD}}$, their requirements for supply voltage cannot meet simultaneously. We employ the dual-supply LDO to solve this problem. By separately supplying the control stage and the power stage of the LDO, we expand the operating range of $V_{\mathrm{A}}$, which supplies the power stage in this design, and reduce the dropout of the LDO to improve the conversion efficiency while maintaining the LDO's normal operation. As shown in Fig. 6, the dualsupply LDO has a wider regulation range compared with the conventional LDO. Note that both of them use NMOS as power transistor, and the supply voltage of dual-supply LDO control stage remains $1.2 \mathrm{~V}$ during simulation. Moreover, the output voltage and the load current are $0.6 \mathrm{~V}$ and $6 \mathrm{~mA}$, respectively. 


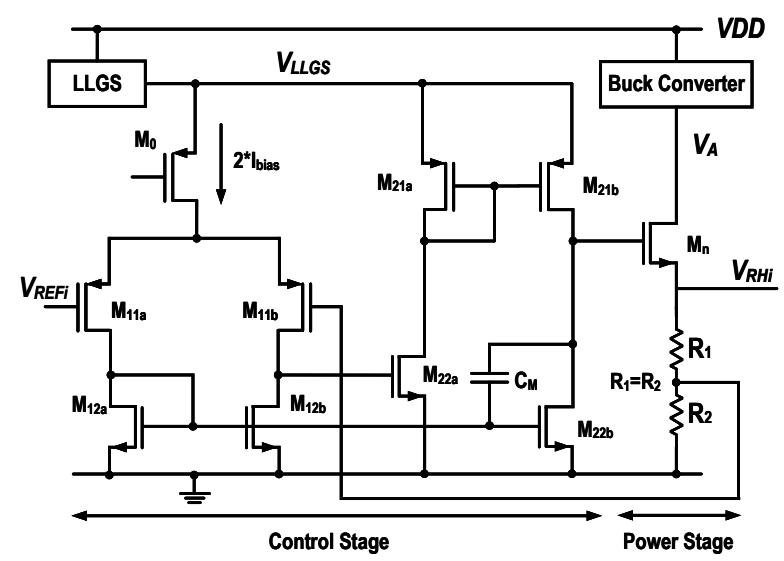

Fig. 5. Schematic of the dual-supply LDO.

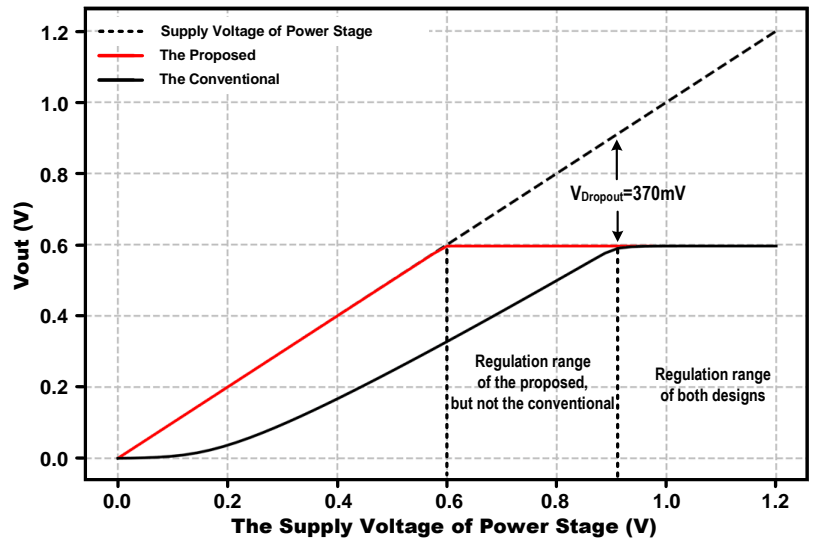

Fig. 6. Input-output characteristic of the proposed dual-supply LDO and the conventional LDO.

The supply noise on the output voltage needs to be considered, especially for the LDO supplied by a dynamically changing voltage. In this design, NMOS is adopted instead of PMOS as the power transistor for a higher PSR. Fig. 7 compares the PSR of dual-supply LDO using NMOS and PMOS power transistors. $V_{\mathrm{RH}}$ and $V_{\mathrm{A}}$ are $0.6 \mathrm{~V}$ and $0.63 \mathrm{~V}$, respectively. The load current is $6 \mathrm{~mA}$. In the frequency range of $1 \mathrm{kHz}$ to $100 \mathrm{kHz}$, which is usually the typical bandwidth of thermal-optic tuning and the fluctuation range of $V_{\mathrm{A}}$, the PSR using NMOS is at least $12 \mathrm{~dB}$ higher than that using PMOS. However, the conventional LDO with NMOS power transistor usually has a relatively large dropout and a narrow output swing, which is undesirable for thermo-optic tuning. We introduce LLGS to solve this problem.

\section{Lightweight Local Generated Supply}

We introduce the LLGS structure in [33] to supply eight LDOs' control stages simultaneously. There are two LLGSs employed, and each supplies half of the control stages in this design. The LLGS provides a sufficiently high local voltage $V_{\text {LLGS }}$ for the control stage, which helps to alleviate the limitation of the NMOS power transistor on LDOs' output swing.

Fig. 8 shows the schematic of the LLGS structure. It includes an error amplifier, a power transistor $\mathrm{M}_{00}$, and a

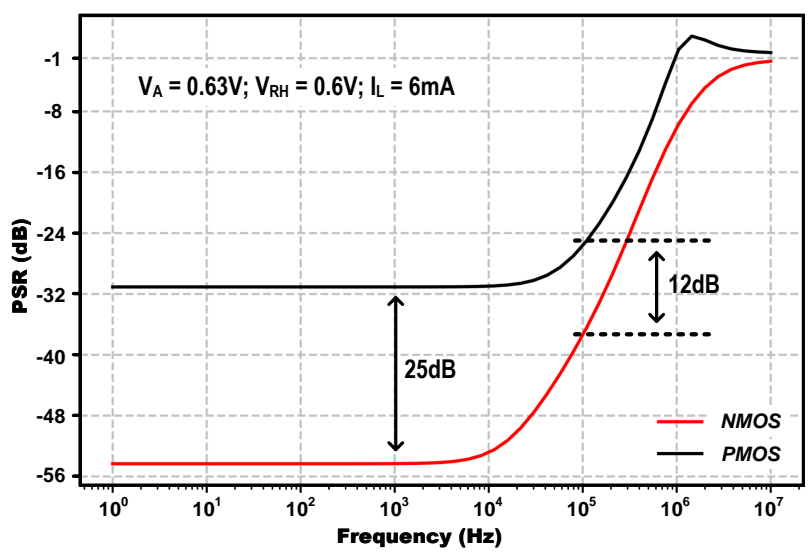

Fig. 7. PSR of the dual-supply LDO with the NMOS and the PMOS power transistors.

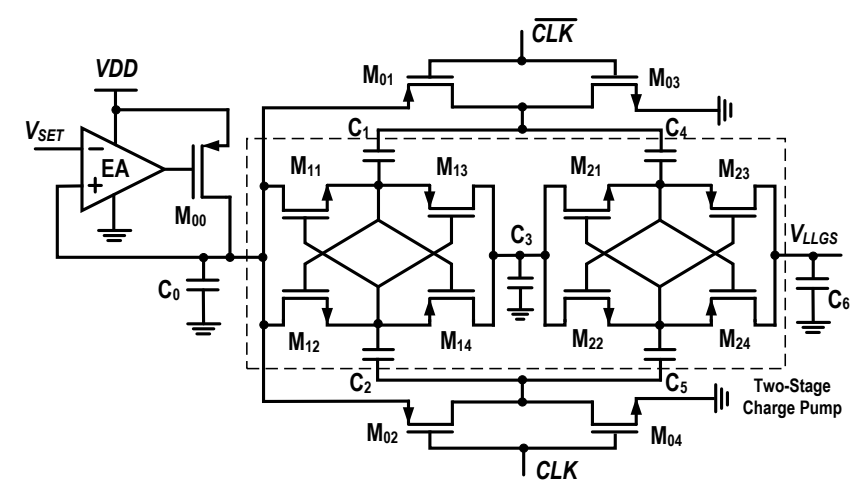

Fig. 8. Implementation of the lightweight local generated supply.

two-stage charge pump. The charge pump doubles its input voltage to supply the LDO's control stage. However, the output voltage of the charge pump can be even higher than the safety voltage of the transistor if $V_{\mathrm{DD}}$ is connected with the input of the charge pump directly. To solve this problem, the LLGS employs a source follower at the input of the charge pump. The output voltage $V_{\text {LLGS }}$ is mainly determined by $V_{\mathrm{SET}}$ and has nothing to do with $V_{\mathrm{DD}}$. By adjusting the $V_{\mathrm{SET}}$, an appropriate $V_{\text {LLGS }}$ for the control stage is obtained. Meanwhile, as the control stage in each dual-supply LDO consumes little quiescent current, the size of $\mathrm{M}_{00}$ can be very small.

We simulate the total quiescent current consumed by a LLGS and the LDOs' control stages it supplies in Fig. 9. In this specific design, the total quiescent current consumed by two LLGSs and eight LDOs' control stages is at most $155 \mu \mathrm{A}$.

\section{Envelope Processing Module}

The envelope processing module is to generate an appropriate $V_{\text {Env }}$ for the switching converter. Fig. 10 shows the structure of the envelope processing module. It consists of a maximum voltage selector and a non-inverting adder. The input signal $V_{\mathrm{REF} 1}$ through $V_{\mathrm{REF} 8}$ are the reference signals generated by the controller in Fig. 1. $V_{\text {Env_REF }}$ is the envelope signal of all input signals, which is obtained through maximum voltage selection. $V_{\text {Offset }}$ is a DC offset that can be adjusted by 


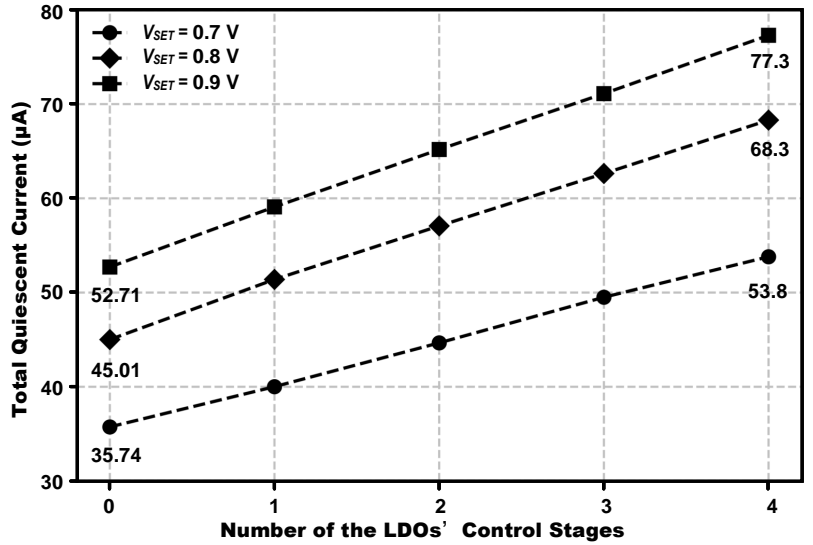

Fig. 9. Quiescent current consumed by a LLGS and the LDOs' control stages supplied by the LLGS.

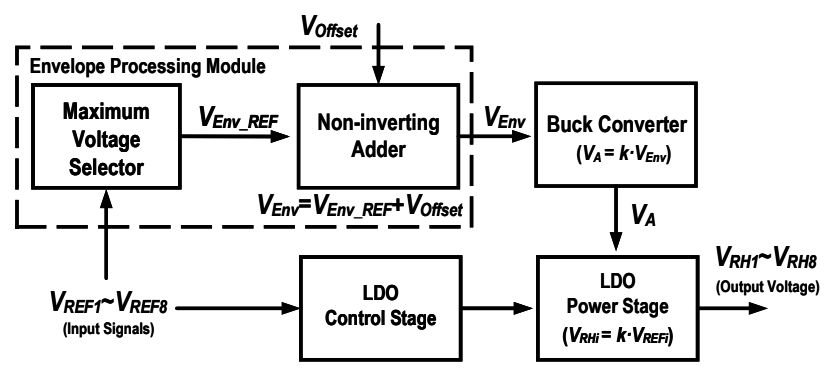

Fig. 10. Structure of the envelope processing module.

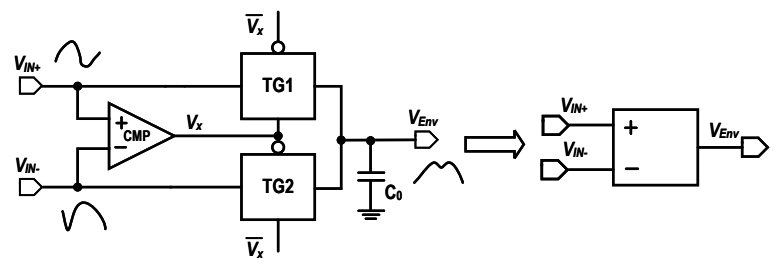

Fig. 11. A simple dual-input maximum voltage selector.

the users, we adopt the non-inverting adder to superimpose the $V_{\text {Offset }}$ on the $V_{\text {Env_REF. The }} k$ is the gain factor of the LDO and the switching converter, which equals 2 in this design. The output voltage $V_{\text {Env }}$ of the envelope processing module is given by

$$
V_{\text {Env }}=V_{\text {Env_REF }}+V_{\text {Offset }} .
$$

Since $V_{\mathrm{RHi}}=k \cdot V_{\mathrm{REFi}}(\mathrm{i}=1,2, \ldots, 8)$, the envelope voltage $V_{\text {Env_RH }}$ of all $V_{\mathrm{RH}} \mathrm{S}$ is given by

$$
V_{\text {Env_RH }}=k \cdot V_{\text {Env_REF }} \text {. }
$$

Meanwhile, as $V_{\mathrm{A}}=k \cdot V_{\mathrm{Env}}$, the dropout between $V_{\mathrm{A}}$ and $V_{\text {Env_RH }}$ is then given by

$$
V_{\text {dropout }}=V_{\mathrm{A}}-V_{\text {Env_RH }}=k \cdot V_{\text {Offset }} .
$$

As a result, an appropriate dropout is obtained by adjusting the $V_{\text {Offset }}$.

A simple dual-input maximum voltage selector is shown in Fig. 11. As shown in Fig. 12, we achieve the maximum

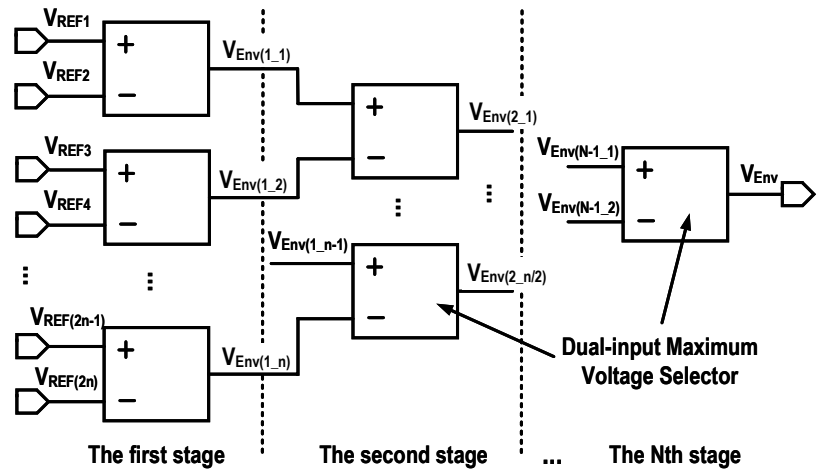

Fig. 12. Cascade multiple dual-input maximum selectors to achieve maximum selection of multiple signals.

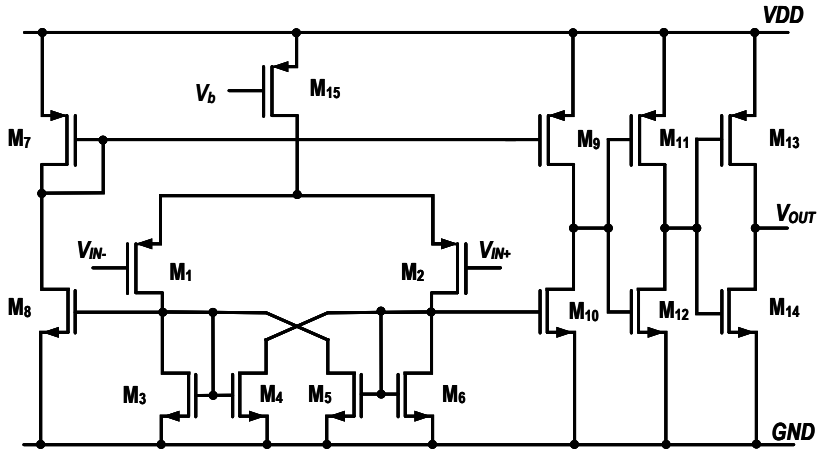

Fig. 13. Schematic of the comparator.

selection of multiple signals by cascading multiple dual-input maximum voltage selectors. The dual-input selector is composed with a comparator and two transmission gates (TGs). According to the result of the comparator, the selector turns on the TG with the higher input signal and turns off the other to generate the envelope of two input signals at its output. When the output voltage of the comparator flips, there will be a brief period that TG1 and TG2 are turned on together, which may introduce glitches at the output of the selector. It is about $13 \mathrm{mV}$ in this design, and the $150 \mathrm{fF}$ capacitor $\mathrm{C}_{0}$ in Fig. 11 is used to decrease these glitches. The comparator, as shown in Fig. 13, is implemented by a source-coupled differential pair with positive feedback to provide a high gain [34]. The gain of the positive feedback gain stage is given by

$$
A_{v}=\sqrt{\frac{\mu_{p}\left(\frac{W}{L}\right)_{1}}{\mu_{n}\left(\frac{W}{L}\right)_{3}}} \frac{1}{1-\alpha},
$$

where $\alpha=(W / L)_{5} /(W / L)_{3}$ is the positive feedback factor. The inverter chains $\mathrm{M}_{11}-\mathrm{M}_{14}$ act as the driver stage such that the transistors $M_{8}$ and $M_{10}$ can be made smaller to reduce the parasitic capacitance at the gates of $\mathrm{M}_{8}$ and $\mathrm{M}_{8}$ for a faster response.

To determine the value of $V_{\text {Offset }}$, we consider the following two non-ideal effects. The first non-ideal effect is the input offset voltage $\Delta V_{\text {os }}$ and the delay time $t_{\text {del_com }}$ of the comparator. Both of them would cause some errors to the output signal of the maximum voltage selector. The maximum error voltage is around $\left(\Delta V_{\text {os }}+\Delta V_{\text {del_com }}\right)$, where $\Delta V_{\text {del_com }}$ is 


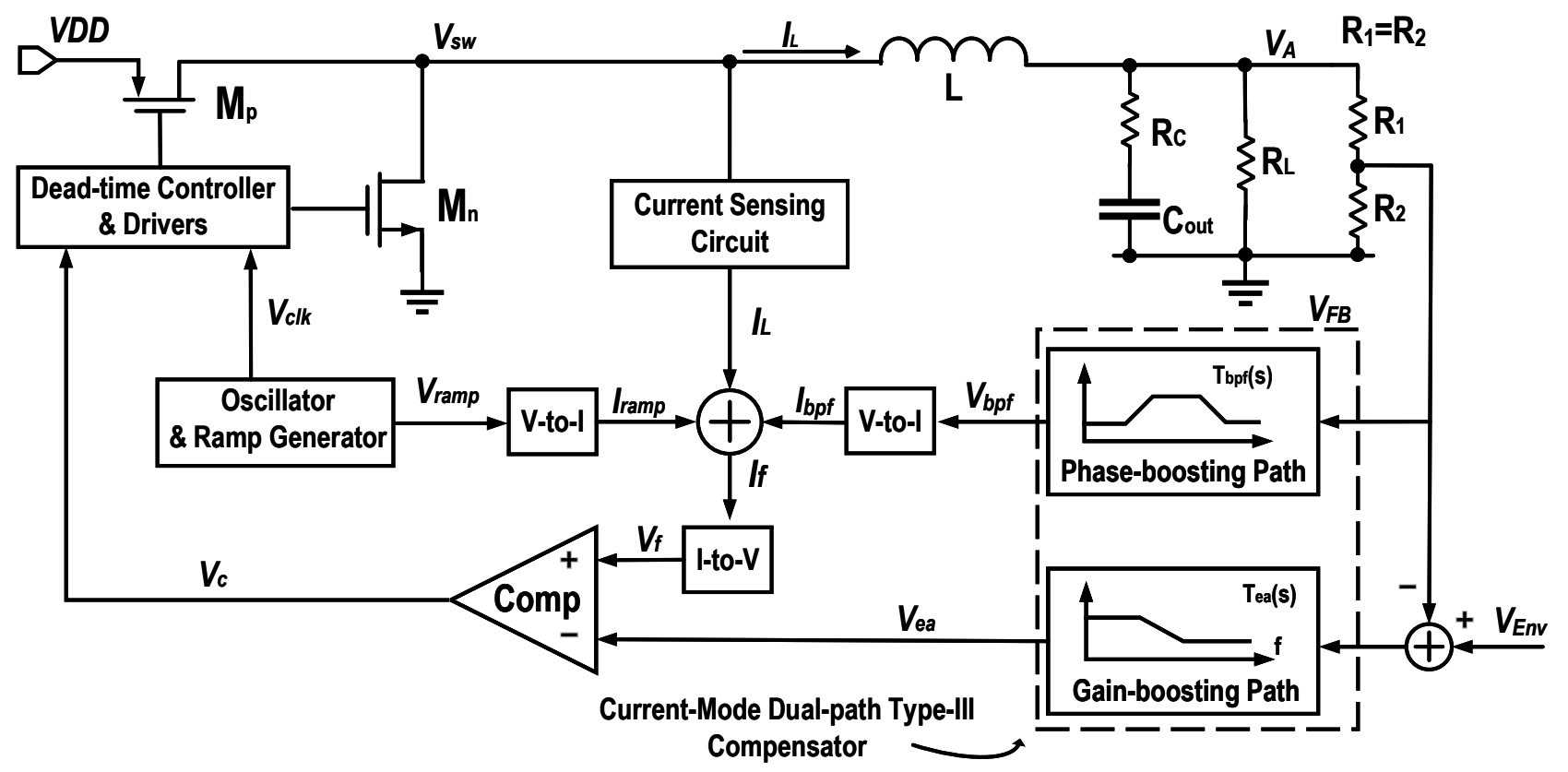

Fig. 14. Structure of current-mode dual-path Type-III compensated buck converter.

the error voltage caused by $t_{\text {del_com. }}$ To avoid the impact of the $\Delta V_{\text {os }}$ and the $\Delta V_{\text {del_com }}$, the minimum $V_{\text {Offset }}$ has to be at least $\left(\Delta V_{\text {os }}+\Delta V_{\text {del_com }}\right)$. For the offset voltage $\Delta V_{\text {os }}$, it is generally several $\mathrm{mV}$ when no offset reduction techniques are utilized [35], and can be further reduced by increasing the area of the input transistors [36]. For these two reasons, the additional DC offset caused by the offset is usually small. For the delay time $t_{\text {del_com }}$, since the simulation results show that the $t_{\text {del_com }}$ is around $15 \mathrm{~ns}$, and the tuning speed for typical thermo-optic tuning is around tens of $\mathrm{kHz}$, the $\Delta V_{\text {del_com }}$ caused by $t_{\text {del_com }}$ can be neglected as well. The second nonideal effect is the delay $t_{\text {del_va }}$ between $V_{\mathrm{A}}$ and $V_{\text {Env RH }}$ as the path to generate $V_{\mathrm{A}}$ is longer than that to generate $V_{\mathrm{RH}} \mathrm{s}$. The simulation results show that the $t_{\text {del_va }}$ in this design is around $110 \mathrm{~ns}$. To avoid the impact of the $t_{\text {del_va }}, V_{\text {Offset }}$ has to reserve some margin. We define the $\Delta V_{\text {del_va }}$ to describe the additional DC offset due to the $t_{\text {del_va. }}$. Since the $\Delta V_{\text {del_va }}$ is related to the frequency and waveform of the specific input signals, the value of $\Delta V_{\text {del_va }}$ is difficult to be analyzed quantitatively. Fortunately, as the tracking speed of most applications based on thermo-optic tuning is relatively slow, $\Delta V_{\text {del_va }}$ is usually small. Besides, adding additional delay units to the shorter path can effectively reduce $t_{\text {del_va }}$ and decrease $\Delta V_{\text {del_va. }}$

After considering these non-ideal effects, the $V_{\text {Offset }}$ we set in the following simulation is around $20 \mathrm{mV}$. The simulation results have verified that this $V_{\text {Offset }}$ is sufficient to cope with the non-ideal effects, and the dropout obtained according to this $V_{\text {Offset }}$ is also small enough to improve the efficiency.

\section{E. Switching Converter}

Compared with the linear regulator, switching converter can maintain sufficiently high conversion efficiency over a wide regulation range. However, due to its relatively large on-chip area and output power, as well as its large off-chip inductor and capacitor, it is not suitable for directly supplying the resistive heaters in large-scale $\mathrm{SiPh}$ integration. Using the proposed architecture can reduce chip area while making full use of the efficiency advantage of the switching converter. For instance, to control a photonic system with 128 optical components, 128 off-chip inductors are required when directly supplying the heaters with switching converters, while the number of the inductors required in the proposed design is only 16 .

The switching converter in proposed design is based on [37], it has been demonstrated in [37] that the buck converter with monolithic current-mode dual-path Type-III compensator has an excellent transient response and a high $f_{\text {ugb }} / f_{\mathrm{sw}}$ ratio compared with recent works in the literature. It can track $180 \mathrm{kHz}$ SC-FDMA waveforms, which is adequate for our application. Fig. 14 shows the structure of this current-mode dual-path Type-III compensated buck converter. In addition, the implementation of the proposed architecture is not limited to specific switching converter, any switching converter with sufficient bandwidth and efficiency can be applied.

\section{F. Evaluation of Efficiency}

The simulation results show that the current consumed by the envelope processing module is around $82 \mu \mathrm{A}$. Since the total load current of eight channels ranges from $20 \mathrm{~mA}$ to $84 \mathrm{~mA}$, the power dissipation of the envelope processing module is negligible. Ignoring the power dissipation of this block can simplify the analysis and will not affect the conclusion.

The $\eta_{\mathrm{ch}_{-} \mathrm{i}}$ is defined as the conversion efficiency of a single channel $\mathrm{i}(\mathrm{i}=1,2, \ldots, 8)$, and it is given by

$$
\begin{aligned}
\eta_{\text {ch_i }} & =\eta_{\text {con }} \cdot \eta_{\text {ldo_i }} \\
& =\eta_{\text {con }} \cdot \frac{I_{\text {load }} V_{\text {RHi }}}{I_{\text {load }} V_{\mathrm{A}}+I_{\text {avg }} V_{\mathrm{DD}}},
\end{aligned}
$$


where $\eta_{\text {con }}$ and $\eta_{\text {ldo_i }}$ are the efficiency of the switching converter, and the channel i of the LDOs, respectively; $I_{\text {load }}$ and $V_{\mathrm{RHi}}$ are the load current and the output voltage of the same channel; $I_{\text {avg }}$ is the average current consumed by a single LDO's control stage, and $I_{\text {avg }}=I_{\text {total }} / N$, where $I_{\text {total }}$ is the total current consumed by LLGSs and all LDOs' control stages, and $N$ is the number of the channels. As illustrated in Fig. 9, the $I_{\text {total }}$ consumed by system is at most $155 \mu \mathrm{A}$, and the power dissipation of the LLGSs and all LDOs' control stages can be neglected as well. Therefore, the $\eta_{\mathrm{ch}_{-} \mathrm{i}}$ can be approximated as

$$
\eta_{\text {ch } \_\mathrm{i}}=\eta_{\text {con }} \cdot \frac{V_{\mathrm{RHi}}}{V_{\mathrm{A}}}
$$

The efficiency of a single channel is also related to the output voltage difference of all channels. Since the multiple channels share the same intermediate voltage $V_{\mathrm{A}}$, it is impossible for the architecture to individually adjust the dropout of each linear power stage. When the output voltages of multiple channels are close to each other, the dropout of every channel is small and the efficiency improvement is obvious; when the output voltage of one channel among them is quite higher than that of other channels, the dropout of the channel with the lowest output voltage is relatively large, and the efficiency improvement of this channel is limited. For this reason, the proposed architecture has high system efficiency for applications where the tracking signals of several adjacent channels are close to each other, which has been discussed in Section III.

The worst-case efficiency as a function of dropout voltage is also shown in Fig. 15. In this case, the output voltage of one channel is higher than that of other channels, while the output voltages of seven other channels are the same. Therefore, except for the channel with higher output voltage, the efficiency improvement of seven other channels is limited. We simulate the conversion efficiency of these seven channels and show the results in Fig. 15. The power consumption of all blocks, including LLGS and the envelope processing module, has been considered when calculating efficiency. The load is $100 \Omega$, and the $\Delta V$ is the voltage difference between the channel with the higher output voltage and seven other channels. Since the output voltage range of this design is 0.25 $1.05 \mathrm{~V}$, the maximum $\Delta V$ is $0.8 \mathrm{~V}$. The $\mathrm{X}$-axis in Fig. 15 is the dropout between $V_{\mathrm{DD}}=1.2 \mathrm{~V}$ and the output voltage. As shown in Fig. 15, the worst-case efficiency of the proposed design is always higher than the theoretical efficiency of the conventional design under the same dropout between $V_{\mathrm{DD}}$ and the output voltage. When $\Delta V$ equals 0 , the output voltages of all eight channels are the same, and the efficiency improvement is obvious compared with the conventional design. As the efficiency of the proposed design is related to $\eta_{\text {con }}$ as well, higher system efficiency is expected by further improving the efficiency of the switching converter.

\section{DESIGN VERIFICATION}

The proposed hybrid dynamic regulator has been implemented in a $130 \mathrm{~nm}$ CMOS process, and Fig. 16 shows the

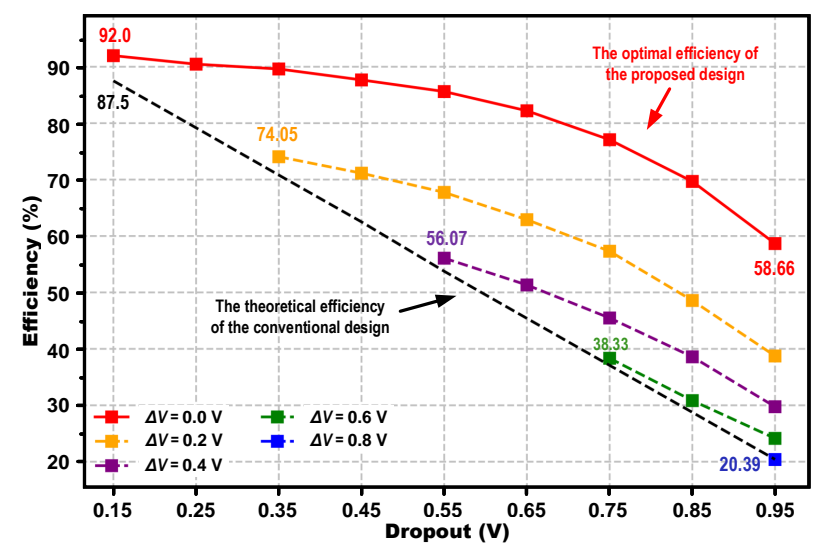

Fig. 15. Worst-case conversion efficiency as a function of dropout voltage.

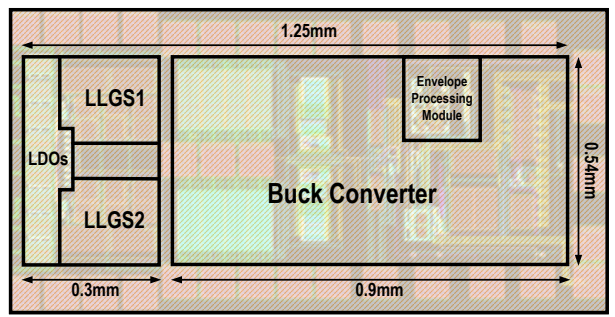

Fig. 16. Layout of the proposed design.

layout of the proposed design with a total area of $0.675 \mathrm{~mm}^{2}$. Extensive post-layout simulation results have been carried out to verify the effectiveness of the proposed architecture. Fig. 17 shows the key waveforms when tracking sinusoidal signals. $V_{\mathrm{REF} 1}$ through $V_{\mathrm{REF} 4}$ are $50 \mathrm{kHz} 0.4 \mathrm{~V}_{\mathrm{pp}}, 40 \mathrm{kHz}$ $0.3 \mathrm{~V}_{\mathrm{pp}}, 30 \mathrm{kHz} 0.2 \mathrm{~V}_{\mathrm{pp}}$, and $20 \mathrm{kHz} 0.1 \mathrm{~V}_{\mathrm{pp}}$ sinusoidal signals respectively. Except for the initial phase, the $V_{\text {REF5 }}$ through $V_{\mathrm{REF} 8}$ are the same as $V_{\mathrm{REF} 1}$ through $V_{\mathrm{REF} 4}$. Fig. 18 shows the key waveforms when tracking triangle signals. $V_{\text {REF1 }}$ through $V_{\text {REF4 }}$, and $V_{\text {REF5 }}$ through $V_{\text {REF8 }}$ are $50 \mathrm{kHz}$ $0.4 \mathrm{~V}_{\mathrm{pp}}, 25 \mathrm{kHz} 0.3 \mathrm{~V}_{\mathrm{pp}}, 40 \mathrm{kHz} 0.4 \mathrm{~V}_{\mathrm{pp}}$, and $20 \mathrm{kHz}$ $0.6 \mathrm{~V}_{\mathrm{pp}}$ triangle signals respectively. The only difference between $V_{\text {REF5 }}$ through $V_{\text {REF8 }}$ and $V_{\text {REF1 }}$ through $V_{\text {REF4 }}$ is the initial phase. Compared with the design in [38], the proposed design can simultaneously regulate more channels, and has a wider tuning range.

The static efficiency when each channel is loaded with the same current is shown in Fig. 19 (a). The peak efficiency is $94.66 \%$ when the output voltage is $1 \mathrm{~V}$ and the load current of each channel is $40 \mathrm{~mA}$. When driving a $100 \Omega$ load, the proposed design achieves around $92 \%$ efficiency at $1 \mathrm{~V}$ output. As shown in Fig. 19 (b), the efficiency of the proposed design is always higher than that of the conventional design, and the efficiency improvement is around 50\% when $V_{\mathrm{RH}}=0.4$ V. The dynamic efficiency when each channel tracks the same sinusoidal signal and drives a $100 \Omega$ load is shown in Fig. 20 (a). The proposed design achieves a dynamic efficiency of around $86 \%$ when tracking $50 \mathrm{kHz}$ sinusoidal singals. Fig. 20 (b) shows that the proposed design achieves over $26 \%$ efficiency improvement compared with the conventional design. Note that the power consumption of all blocks has 


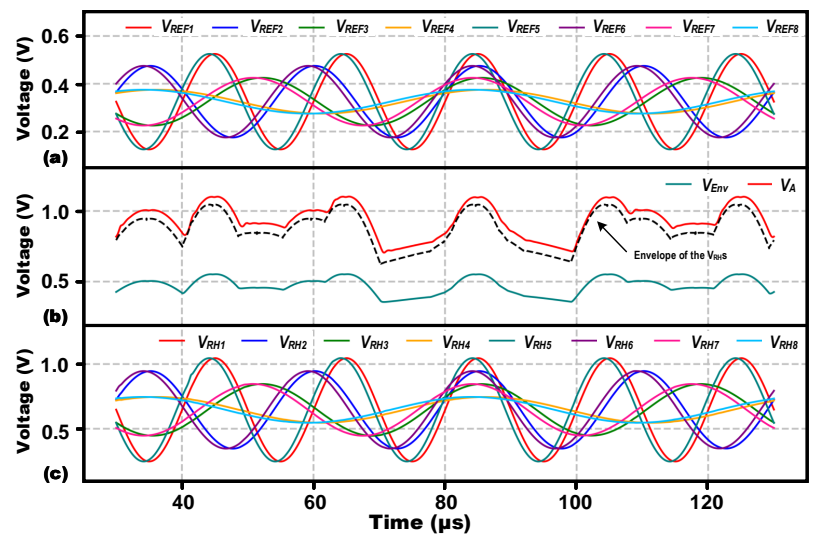

Fig. 17. Key waveforms when tracking sinusoidal signals: (a) $V_{\mathrm{REF} 1}$ through $V_{\mathrm{REF} 8}$; (b) $V_{\text {Env }}$ and $V_{\mathrm{A}}$; (c) $V_{\mathrm{RH} 1}$ through $V_{\mathrm{RH} 8}$.

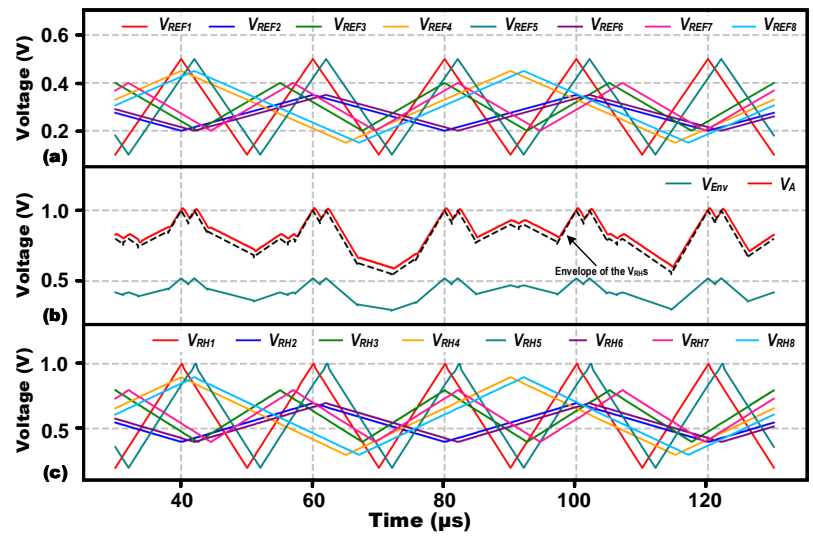

Fig. 18. Key waveforms when tracking triangle signals: (a) $V_{\mathrm{REF} 1}$ through $V_{\mathrm{REF} 8}$; (b) $V_{\text {Env }}$ and $V_{\mathrm{A}}$; (c) $V_{\mathrm{RH} 1}$ through $V_{\mathrm{RH} 8}$.

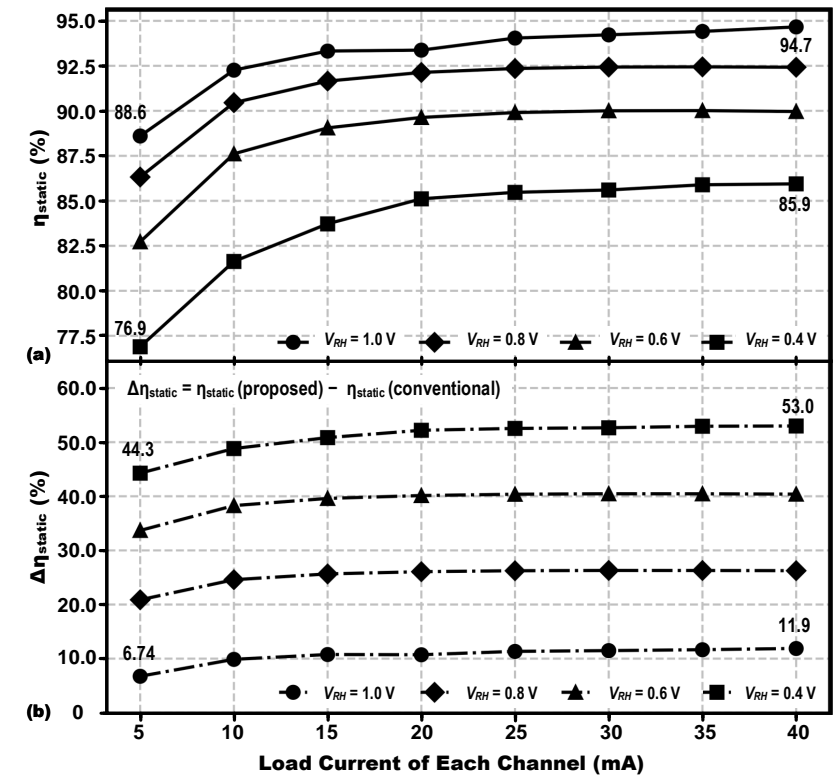

Fig. 19. (a) Static efficiency and (b) $\Delta \eta_{\text {static }}$ as a function of load current.

been considered when calculating the static and dynamic efficiency, and the dropout between $V_{\mathrm{A}}$ and $V_{\mathrm{RH}}$ is about

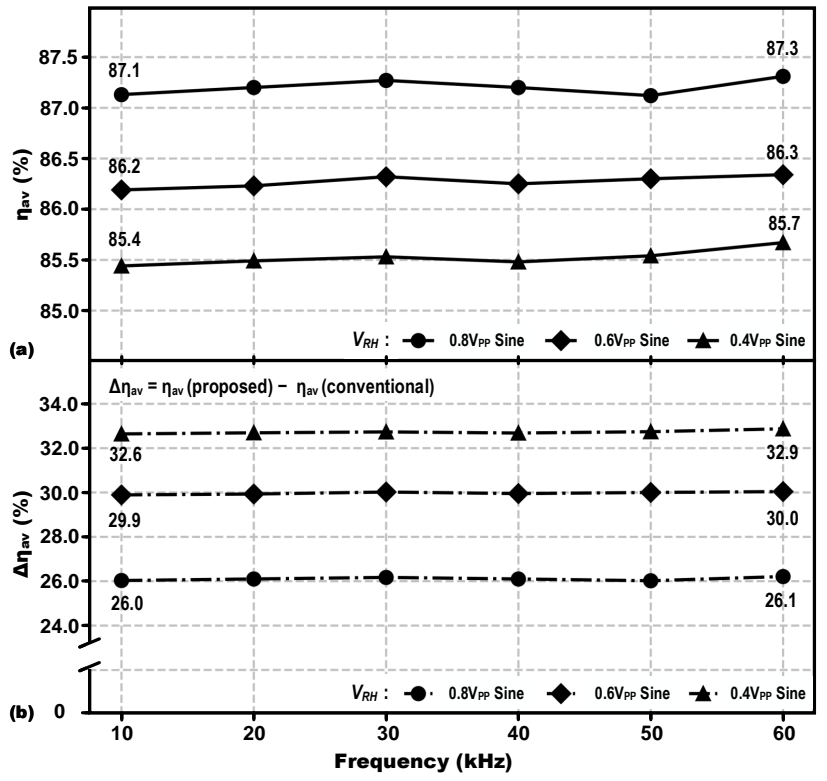

Fig. 20. (a) Dynamic efficiency and (b) $\Delta \eta_{\text {av }}$ when tracking sinusoidal signals at different frequencies.

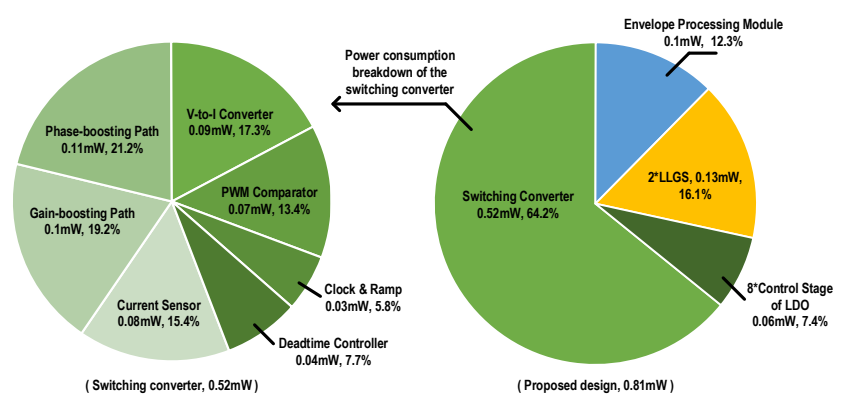

Fig. 21. Quiescent power consumption breakdown.

TABLE II

PERFoRMANCE SumMARY OF THE Proposed DESIGN

\begin{tabular}{cc}
\hline Application & Thermo-Optic Tuning \\
Tech.(nm) & 130 \\
Channels & 8 \\
Supply Voltage(V) & 1.2 \\
Output Voltage(V) & $0.25-1.05$ \\
Total Quiescent Current( $\mu \mathrm{A})$ & 668 \\
Peak Efficiency & $\mathbf{9 4 . 7 \%}$ \\
Dynamic Efficiency & $\mathbf{8 6 \%} @ 50 \mathrm{kHz}$ Sine \\
Tracking Speed & $\mathbf{5 0 k H z}$ (sinusoidal) \\
Load Resistor $(\mathbf{\Omega})$ & 100 \\
Chip Area(mm $\left.{ }^{2}\right)$ & $\mathbf{0 . 6 7 5}$ \\
Average Area Per Ch. $\left(\mathrm{mm}^{2}\right)$ & $\mathbf{0 . 0 8 4}$ \\
\hline
\end{tabular}

$40 \mathrm{mV}$. The quiescent power consumption breakdown of the proposed design is shown in Fig. 21. The power dissipation of two LLGSs, eight control stages, and the envelope processing module is around $0.3 \mathrm{~mW}$

TABLE II provides the performance summary of the proposed hybrid dynamic regulator. To the best of our knowledge, it is the first time that dynamic supply modulation is introduced for thermo-optic tuning, which has different requirements 
compared with the previous RF/AF PA dynamic supply case. As previous designs are targeted for different applications, there are no appropriate existing works to be compared with.

\section{CONCLUSION}

In this paper, we have presented a novel multi-channel switching-linear hybrid dynamic regulator architecture with dual-supply LDOs for large-scale thermo-optic tuning. Compared with conventional designs, the proposed architecture reduces the LDO dropout voltage and increases the conversion efficiency through dynamic supply modulation. The efficiency improvement is particularly effective when tracking signals of several adjacent channels are close to each other, e.g., the wavelength tuning of a microring array. The proposed architecture can be implemented using different switching converters and LDOs. A prototype with post-layout simulation results has been used to verify the advantages of the proposed architecture.

\section{REFERENCES}

[1] A. H. Atabaki et al., "Integrating photonics with silicon nanoelectronics for the next generation of systems on a chip," Nature, vol. 556, no. 7701, pp. 349-354, 2018.

[2] M. Tan, K. Ye, D. Ming, Y. Wang, and J. Feng, "Towards electronicphotonic-converged thermo-optic feedback tuning," Journal of Semiconductors, vol. 42, no. 2, p. 023104, 2021.

[3] S. Jie, T. Erman, Y. Ami, H. Ehsan Shah et al., "Large-scale nanophotonic phased array," Nature, vol. 493, no. 7431, pp. 195-199, 2018

[4] S. Chung, H. Abediasl, and H. Hashemi, "A monolithically integrated large-scale optical phased array in silicon-on-insulator CMOS," IEEE Journal of Solid-State Circuits, vol. 53, no. 1, pp. 275-296, 2018.

[5] X. Qiang, X. Zhou, J. Wang, C. M. Wilkes, L. Thomas, O. Sean, K. Laurent, G. D. Marshall, S. Raffaele, and T. C. Ralph, "Large-scale silicon quantum photonics implementing arbitrary two-qubit processing," Nature Photonics, vol. 12, no. 9, pp. 543-549, 2018.

[6] J. Wang et al., "Multidimensional quantum entanglement with largescale integrated optics," Science, vol. 360, no. 6386, 2018.

[7] Y. Shen et al., "Deep learning with coherent nanophotonic circuits," Nature Photonics, pp. 441-446, 2017.

[8] A. Ribeiro, S. Declercq, U. Khan, M. Wang, L. V. Iseghem, and W. Bogaerts, "Column-row addressing of thermo-optic phase shifters for controlling large silicon photonic circuits," IEEE Journal of Selected Topics in Quantum Electronics, vol. 26, no. 5, pp. 1-8, 2020.

[9] S. A. Miller et al., "512-element actively steered silicon phased array for low-power LIDAR." Optical Society of America, 2018, p. JTh5C.2.

[10] H. Abediasl and H. Hashemi, "Monolithic optical phased-array transceiver in a standard SOI CMOS process," Opt. Express, vol. 23 , no. 5, pp. 6509-6519, Mar 2015.

[11] C. Sun et al., "A $45 \mathrm{~nm}$ CMOS-SOI monolithic photonics platform with bit-statistics-based resonant microring thermal tuning," IEEE Journal of Solid-State Circuits, vol. 51, no. 4, pp. 893-907, 2016.

[12] S. Agarwal, M. Ingels, M. Pantouvaki, M. Steyaert, P. Absil, and J. Van Campenhout, "Wavelength locking of a Si ring modulator using an integrated drop-port OMA monitoring circuit," IEEE Journal of SolidState Circuits, vol. 51, no. 10, pp. 2328-2344, 2016.

[13] M.-H. Kim et al., "A temperature controller IC for maximizing Si microring modulator optical modulation amplitude," Journal of Lightwave Technology, vol. 37, no. 4, pp. 1200-1206, 2019.

[14] R. Fatemi, A. Khachaturian, and A. Hajimiri, "A nonuniform sparse 2-D large-FOV optical phased array with a low-power PWM drive," IEEE Journal of Solid-State Circuits, vol. 54, no. 5, pp. 1200-1215, 2019.

[15] M. Hassan, L. E. Larson, V. W. Leung, and P. M. Asbeck, "A combined series-parallel hybrid envelope amplifier for envelope tracking mobile terminal RF power amplifier applications," IEEE Journal of Solid-State Circuits, vol. 47, no. 5, pp. 1185-1198, 2012.

[16] T.-W. Kwak, M.-C. Lee, B.-K. Choi, H.-P. Le, and G.-H. Cho, "A 2W CMOS hybrid switching amplitude modulator for EDGE polar transmitters," in 2007 IEEE International Solid-State Circuits Conference. Digest of Technical Papers, 2007, pp. 518-619.
[17] M. Tan and W.-H. Ki, "A $100 \mathrm{MHz}$ hybrid supply modulator with ripplecurrent-based PWM control," IEEE Journal of Solid-State Circuits, vol. 52, no. 2, pp. 569-578, 2017.

[18] W.-Y. Chu, B. Bakkaloglu, and S. Kiaei, "A $10 \mathrm{MHz}$ bandwidth, $2 \mathrm{mV}$ ripple PA regulator for CDMA transmitters," IEEE Journal of Solid-State Circuits, vol. 43, no. 12, pp. 2809-2819, 2008.

[19] R. Bakker and M. Duffy, "Improving the efficiency of class-D audio amplifier systems using envelope tracking DC-DC power supplies," in 2018 IEEE Applied Power Electronics Conference and Exposition (APEC), 2018, pp. 412-418.

[20] S. Chung, M. Nakai, and H. Hashemi, "Low-power thermo-optic silicon modulator for large-scale photonic integrated systems," Opt. Express, vol. 27, no. 9, pp. 13430-13459, Apr 2019.

[21] S. A. Miller et al., "Large-scale optical phased array using a low-power multi-pass silicon photonic platform: supplementary material," Optica, vol. 7 , no. 1 , p. 3, 2020 .

[22] D. Celo et al., "Thermo-optic silicon photonics with low power and extreme resilience to over-drive," in 2016 IEEE Optical Interconnects Conference (OI), 2016, pp. 26-27.

[23] P. Dong, W. Qian et al., "Thermally tunable silicon racetrack resonators with ultralow tuning power," Opt. Express, vol. 18, no. 19, pp. 20298 20 304, Sep 2010.

[24] P. Dong, W. Qian, H. Liang et al., "Low power and compact reconfigurable multiplexing devices based on silicon microring resonators," Opt. Express, vol. 18, no. 10, pp. 9852-9858, May 2010.

[25] M. Bahadori et al., "Thermal rectification of integrated microheaters for microring resonators in silicon photonics platform," J. Lightwave Technol., vol. 36, no. 3, pp. 773-788, Feb 2018

[26] H. Jayatilleka, K. Murray, M. Ángel Guillén-Torres, M. Caverley, R. Hu, N. A. F. Jaeger, L. Chrostowski, and S. Shekhar, "Wavelength tuning and stabilization of microring-based filters using silicon in-resonator photoconductive heaters," Opt. Express, vol. 23, no. 19, pp. 25084 25 097, Sep 2015.

[27] S. K. Selvaraja, W. Bogaerts, P. Dumon, D. Van Thourhout, and R. Baets, "Subnanometer linewidth uniformity in silicon nanophotonic waveguide devices using CMOS fabrication technology," IEEE Journal of Selected Topics in Quantum Electronics, vol. 16, no. 1, pp. 316-324, 2010.

[28] L. Chrostowski, X. Wang, J. Flueckiger, Y. Wu, Y. Wang, and S. T. Fard, "Impact of fabrication non-uniformity on chip-scale silicon photonic integrated circuits," in OFC 2014, 2014, pp. 1-3.

[29] M. S. Nawrocka, T. Liu, X. Wang, and R. R. Panepucci, "Tunable silicon microring resonator with wide free spectral range," Appl.phys.lett, vol. 89, no. 7, pp. 1081-393, 2006.

[30] P. Dong, R. Shafiiha et al., "Wavelength-tunable silicon microring modulator," Opt. Express, vol. 18, no. 11, pp. 10 941-10 946, May 2010.

[31] P. Verheyen et al., "Highly uniform $28 \mathrm{~Gb} / \mathrm{s} \mathrm{Si}$ photonics platform for high-density, low-power WDM optical interconnects," 2014, p. IW3A.4.

[32] M. Tan and W.-H. Ki, "Current-mirror miller compensation: An improved frequency compensation technique for two-stage amplifiers," in 2013 International Symposium on VLSI Design, Automation, and Test (VLSI-DAT), 2013, pp. 1-4.

[33] C. Yang, K. Ye, and M. Tan, "A 0.5-V capless LDO with 30-dB PSRR at $10-\mathrm{kHz}$ using a lightweight local generated supply," IEEE Transactions on Circuits and Systems II: Express Briefs, vol. 67, no. 10, pp. 17851789,2020

[34] R. Gregorian, Introduction to CMOS Op-Amps and Comparators. New York: Wiley, 1999.

[35] J. Atherton and H. Simmonds, "An offset reduction technique for use with CMOS integrated comparators and amplifiers," IEEE Journal of Solid-State Circuits, vol. 27, no. 8, pp. 1168-1175, 1992.

[36] A. Khorami, M. B. Dastjerdi, and A. F. Ahmadi, "A low-power highspeed comparator for analog to digital converters," in 2016 IEEE International Symposium on Circuits and Systems (ISCAS), 2016, pp. 2010-2013.

[37] Y. Zhang and M. Tan, "A buck converter using a fully-integrated currentmode dual-path Type-III compensator for NB-IoT applications," in 2018 IEEE International Conference on Integrated Circuits, Technologies and Applications (ICTA), 2018, pp. 47-48.

[38] S. Zhang, Z. Wang, and M. Tan, "A dual-channel switching-linear series-connected hybrid dynamic power supply with dual-supply LDOs for thermo-optical tuning," in 2021 IEEE International Conference on Integrated Circuits, Technologies and Applications (ICTA), 2021, pp. 143-144. 